\title{
Effectiveness of Take-Home Naloxone Programs in Athletic Training: An Evidence-to-Practice Review
}

\author{
Michael J. Palm, MS, SCAT, ATC; Amanda N. Flanscha, MS, SCAT, ATC; Zachary K. Winkelmann, PhD,
} SCAT, ATC

University of South Carolina, Columbia, SC

\begin{abstract}
The number of opioid overdoses (ODs) has risen in recent years and has become more complex due to the coinvolvement of both prescription and illicit opioid drug use. Provisional programs for take-home naloxone (a medication designed to rapidly reverse opiate OD symptoms) kits have been distributed to combat this potentially fatal epidemic. Although there is strong evidence to support the efficacy of naloxone in the reversal of opiate $O D$, there is limited evidence to support the efficacy of take-home naloxone (THN) kits. The purpose of this evidence-to-practice review was to summarize a systematic review on the efficacy of THN programs. The authors aimed to include studies of THN programs that both trained opioid users in OD prevention and reported on OD outcomes. The Bradford Hill criteria (strength of association, temporality, consistency, specificity, dose-response relationship, biological plausibility, coherence, experimental evidence, and analogy) and five additional criteria (measure cost-effectiveness, absence of negative consequences, feasibility of implementation/expansion/ coverage, unanticipated benefits, and special populations) was used as dependent variables to determine the impact of public health intervention where randomized control trials (RCTs) are not ethically feasible or operationally practical. All 22 studies included provided empirical support using the Bradford Hill Criteria for community based THN programs. Despite being unable to deduce whether death would have occurred without the administration of THN, the studies combined accounted for an estimated 2316 successful opioid OD reversals. Thus, there is a strong association between THN administration and overdose survival. Additionally, there was a low rate of adverse events: withdrawal symptoms $(2.8 \%)$, vomiting $(2.2 \%)$, agitation $(2.1 \%)$, seizures $(0.1 \%)$. Consequently, we recommend that athletic trainers include opioid crisis management equipment and procedures in a site-specific policies manual. Clinical relevance is highly dependent on patient population and geographic location, considering $90 \%$ of reversed ODs were heroin induced. Application to individuals in organized sport is minimal, but nonetheless, individuals who are prescribed opioids for pain management should be candidates for THN programs. Athletic trainers and guardians of minors prescribed opioid medications should be educated on dispensing medication, best practices for opioid crisis management, and distribution of naloxone/THN.
\end{abstract}

Key Phrases

Public health, patient education, triage and emergency care

\section{Correspondence}

Dr. Zachary Winkelmann, 1300 Wheat Street, Columbia, SC 29208.

E-mail:winkelz@mailbox.sc.edu

Twitter: @zachwinkelmann

\section{Full Citation}

Palm MJ, Flanscha AN, Winkelmann ZK. Effectiveness of take-home naloxone programs in athletic training: An evidence-to-practice review. Clin Pract Athl Train. $2021 ; 4(2)$ : 26-32.

https://doi.org/10.31622/2021/0004.2.4.

Submitted: April 17, 2020 Accepted: January 22, 2021.

\section{ORIGINAL REFERENCE}

McDonald $R$, Strang J. Are take-home naloxone programs effective? Systematic review utilizing application of the Bradford Hill criteria. Addiction. 2016;111(7):1177-1187. doi: 10.1111 /add.13326.

\section{SUMMARY}

\section{CLINICAL PROBLEM AND QUESTION}

$E$ rom $1999-2017,56.8 \%$ of the 702,568 drug overdose (OD) deaths in the United States involved opioids. ${ }^{1}$ The number of opioid ODs has risen in recent years, and combating the epidemic has become more complex due to the coinvolvement of both prescription opioid and illicit opioid (e.g. heroin, illicit fentanyl) drug use. ${ }^{2}$ Opioid ODs can be reversed and lives saved with the timely administration of naloxone. ${ }^{3,4}$ Naloxone is a mu-opiate antagonist that rapidly reverses opiate-induced respiratory depression. ${ }^{3}$ A provisional program for take-home naloxone (THN) kits was first introduced in 1996.5 These THN programs typically involve training drug users and/or family members and peers on risk 
awareness, emergency management, and naloxone administration. Both the World Health Organization $(\mathrm{WHO})^{3}$ and the U.S. Surgeon Generalo have released statements emphasizing the importance of '[opioid users], health care practitioners, family and friends of people who have an opioid use disorder, and community members who come into contact with people at risk for opioid OD, knowing how to use naloxone and keeping it within reach.'6 As such, athletic trainers are healthcare providers and should be trained on the identification and management of opioid crises. Adolescents who participated in sports were found to have approximately $17 \%$ increased odds of non-medical opioid use as compared to peers who did not participate in sports. 7 When looking across sport-specific use, adolescents who participated in football or wrestling had a $50 \%$ increased risk of nonmedical opioid use as compared to peers who did not participate in sports. 7 Among elite athletes, opioids are one of the most used substances. ${ }^{8}$

Athletic trainers are recommended to educate and train those at risk of opioid OD on use of THNs. If resources are not available in the athletic training facility, it is recommended that patients be educated on options available to obtain THNs and other counseling services. The purpose of this guiding systematic review was to answer the clinical question: are take home naloxone programs effective? The primary purpose of this evidenceto-practice review was to examine the applicability of THNs in athletic training clinical practice.

\section{SUMMARY OF LITERATURE}

A systematic review was conducted to find the effectiveness of THN programs. The electronic databases searched included Medline, Psychlnfo (both via OVID), and PubMed. The following Boolean search query was used: (opioid OR opiate) AND overdose AND prevention. Original quantitative (or mixed method) studies of

Copyright $\left({ }^{\circ}\right.$ by Indiana State University All rights reserved. ISSN Online 2577-8188 randomized or observational trials articles from January 1946 to June 2015 were identified, yielding 1397 articles. To be included in the review, studies were screened using the title and abstract. Studies had to include THN programs that trained opioid users in OD prevention AND reported on $O D$ outcomes. After eliminating duplicates, non-English, and irrelevant articles, 36 papers were found. The exclusion criteria included: 1) case studies, 2) papers that reported on buprenorphine/naloxone, 3) papers that did not report primary research data, and 4) papers that did not report on heroin/opioid users, naloxone, or OD.

Upon final analysis, 22 articles were identified to be used in the systematic review. Data was extracted using the Strengthening the Reporting of Observational Studies in Epidemiology (STROBE) checklist and analyzed for study quality. Fifteen of the 22 articles were conducted in the United States, two in Canada, four in the United Kingdom, and one in both the United Kingdom and Germany, providing a geographically diverse population. There was a large variation between studies in terms of size $(n=24-2912$; median=203) and quality (study quality score $=4-7$ out of 8 ). Authors reported that many articles were more descriptive reports rather than structured study designs. Although these reports were beneficial for communications to other practitioners, these reports lacked structure in design and analytic rigor. Only 9 of the 22 studies included systematic follow-ups with participants after THN administration. Since the sample sizes were so small, follow-up data was not included because it was not representative of all studies, posing a threat to external validity. The inconsistency in study design and reporting posed a threat to the internal validity and as a result, small variances exist within the methods of the studies included. A narrative synthesis was used instead of a meta-analysis for the analysis of the 22 studies. 


\section{SUMMARY OF INTERVENTION}

This systematic review analyzed studies that distributed THN programs. Although RCTs are considered best practice in research, this would not be ethical for determining the effectiveness of THN. Consequently, none of the studies included a control group of individual participants selected to not receive $\mathrm{THN}$. Instead, some studies used community-based control comparison groups, or communities in which THN had not been implemented or offered. Studies were analyzed under the assumption that communities had equal variances. All the studies evaluated were retrospective and examined the overall effectiveness of THN for participants that used it after overdosing. Included studies reported descriptive statistics on the number of THN administrations, overdose reversals, and adverse events. These values were used as a proxy to represent the effectiveness and safety of THN.

\section{SUMMARY OF OUTCOMES}

The authors of the guiding systematic review used the Bradford Hill Criteria and the WHO "Evidence to Action Report" to analyze the included studies. The Bradford Hill Criteria is a list of nine items analyzed to determine causality when only correlational data is available. 9 The nine items (Table 1) included strength of association, temporality, consistency, specificity, doseresponse relationship, biological plausibility, coherence, experimental evidence, and analogy. The Bradford Hill Criteria is often used to assess impact for public health interventions when random control trials are not practicable. ${ }^{9}$ Analysis of five additional criteria were included and related to feasibility and implementation: cost-effectiveness, absence of negative consequences, feasibility of implementation/ expansion/ coverage, unanticipated benefits, and special populations (Table 2). These additional criteria have been valuably applied in a WHO "Evidence to Action" report analyzing the

Copyright $\left({ }^{\circ}\right.$ by Indiana State University All rights reserved. ISSN Online 2577-8188 effectiveness of needle-exchange interventions in reducing HIV.

\section{FINDINGS AND CLINICAL IMPLICATIONS}

All 22 studies analyzed provided empirical evidence to meet all nine of the Bradford Hill criteria in support of THN intervention in nonspecific healthcare settings explained in the following sections. In 17 of the 22 studies reporting THN administrations following an OD, $2336 / 2249(96.3 \%)$ successful OD reversals were reported. This implies a strong association between THN and successful opioid OD reversals.

Studies that lacked control groups make it hard to definitively conclude that OD reversals happened because of THN rather than the body filtering and metabolizing the drug. An interrupted time series analysis (regarded as the strongest design for quasi-experimental research) done in Massachusetts distributed THN programs to 2912 participants across 19 communities. ${ }^{10}$ Each community with a THN program served as its own geographic control prior to implementation, and those without THN served as time controls. This study revealed the temporality of THN and significant reduction in $O D$ mortality in communities that had THN programs.

Consistency for THN programs effectiveness is demonstrated through the stability of OD reversal rates across the various geographical regions that data was collected from. 15 different regions, states and countries provided significant support for the consistency of THN programs. Biological plausibility, known as the biological or pharmacological mechanism to explain the outcome of a treatment, is significant in the therapeutic effect of naloxone. Naloxone is an opioid antagonist that binds to opioid receptors and blocks the effects of the drug. The successful reversal of 2249 opioid ODs across all but one of the analyzed studies shows the strong support of biological plausibility. 
Table 1. Bradford Hill criteria and application to take-home naloxone

\begin{tabular}{|c|c|}
\hline Criterion & Take-home naloxone (THN) \\
\hline Strength of association & How strong is the association between THN and OD reversal? \\
\hline Temporality & Did the distribution of THN precede a reduction in OD deaths? \\
\hline Consistency & $\begin{array}{l}\text { Have there been multiple observations of OD reversals because of THN } \\
\text { provision? }\end{array}$ \\
\hline Specificity & Does THN have the unique effect of reversing opioid ODs? \\
\hline Dose-response relationship & Does increased THN supply go together with more OD reversals? \\
\hline Biological plausibility & $\begin{array}{l}\text { Is it biologically plausible that a reduction in OD deaths occurs when THN is } \\
\text { available? }\end{array}$ \\
\hline Coherence & $\begin{array}{l}\text { Are there documented examples of opioid OD mortality declining without } \\
\text { THN availability? If so, does this empirical evidence conflict with the } \\
\text { assumed association between THN and OD prevention? }\end{array}$ \\
\hline Experimental evidence & $\begin{array}{l}\text { Is there (semi)experimental evidence to support the hypothesized impact of } \\
\text { THN on OD mortality? }\end{array}$ \\
\hline Analogy & Is there a treatment like THN that leads to an outcome like OD reversal? \\
\hline
\end{tabular}

An Australian study demonstrated the decline in opioid OD between 2001 and 2002 before naloxone became available in 2011 . However, 21 studies reported successful reversal rates of opioid OD which contributes to the coherence criteria. The specificity of THN throughout the studies analyzed that naloxone is specifically for opioid reversal and has no effect on those suffering from a cocaine OD or other type of drug OD. All 22 studies reported on heroin use and one with long-acting opioid use, all displaying strong specificity for naloxone and opioid reversal.

Dose-response relationship criteria was only assessed in one of the 22 studies, resulting in only partially fulfilling the criteria. In the Massachusetts study, the 19 communities broken into three categories for THN implementation: zero, low (1100 enrollments) and high (>100 enrollments). Low and high implementation resulted in reduced deaths from ODs compared to communities without implementation, providing limited, but supporting evidence for dose-response impact.

Copyright (C) by Indiana State University All rights reserved. ISSN Online 2577-8188
In athletic training practice, take-home naloxone programs may be compared to other emergency medications such as adrenaline (epinephrine) injection kits for allergic reactions or glucagon for insulin OD. Training in the use of naloxone is also compared to the use of automatic external defibrillators and cardiopulmonary resuscitation for those likely to experience these emergencies regardless of their medical background. Like these, time is critical in administration and thus fulfills the analogy criteria.

The WHO "Evidence to Action" criteria were all fully or partially fulfilled in support of THN programs. Studies conducted in the United States and Russia revealed THN interventions are cost-of opioid ODs). 11,12 Across the studies analyzed in this systematic review, THN interventions had a low number of adverse events. The studies which did report adverse events showed these to be associated more with symptoms of withdrawal rather than due to the naloxone. The implementation of THN programs across several 
Table 2. Feasibility and implementation criteria and application to take-home naloxone

\begin{tabular}{|c|c|}
\hline Criterion & Take-home naloxone (THN) \\
\hline Cost-effectiveness & $\begin{array}{l}\text { Is THN for lay OD reversal cost-effective compared to } \\
\text { treatment as usual (no intervention)? }\end{array}$ \\
\hline Absence of negative consequences & $\begin{array}{l}\text { Does the distribution of THN to users bear the risk of adverse } \\
\text { events? }\end{array}$ \\
\hline $\begin{array}{l}\text { Feasibility of } \\
\text { implementation/expansion/coverage }\end{array}$ & $\begin{array}{l}\text { Is it feasible to introduce THN distribution in diverse settings, } \\
\text { including resource-poor settings, and scale up implementation? }\end{array}$ \\
\hline Unanticipated benefits & $\begin{array}{l}\text { Does the distribution of THN to users lead to unanticipated } \\
\text { benefits? }\end{array}$ \\
\hline Special populations & $\begin{array}{l}\text { How successful are THN programs in reaching special } \\
\text { populations that have been identified as particularly 'at-risk' } \\
\text { opioid users? }\end{array}$ \\
\hline
\end{tabular}

locations and circumstances shows the feasibility of implementation, expansion, and coverage. The rapid expansion of community naloxone distribution programs was observed in San Francisco in the 2000s especially in places with low resources. ${ }^{13}$ Unanticipated benefits were seen in a few studies, ranging from participants entering treatment $(25 \%)$ and decreasing drug use following their OD reversal $(53 \%)$, to participants being tested for comorbid conditions and family members being educated on the use of naloxone (28\%).14 Take-home naloxone programs were implemented in several different opioid populations including patients who were detoxing, the homeless, users of methadone, and the incarcerated. This provides evidence for THN programs to be used in special populations across several settings and various demographic populations.

All these criteria provide support for athletic trainers to implement naloxone or THN programs at their clinical sites as a tertiary prevention strategy, or harm reduction, to opioid OD. Because athletic trainers have experience in the primary and secondary prevention of many health conditions, implementing other opioid OD prevention strategies would not only be feasible but beneficial to combat the public health epidemic within their clinical practice and community. Athletic trainers may do this through

Copyright (C) by Indiana State University All rights reserved. ISSN Online 2577-8188 education and policy development and implementation. Depending on different geographical locations, heroin use may be more prevalent in the community, and organizationwide education on naloxone administration may be valuable. Regardless of location, long-acting opioid use remains of high concern. Athletic populations prescribed these drugs as pain killers are at risk for dependence and $O D$ without the proper education on how to use them. In addition to the education provided by the prescribing practitioner, athletic trainers typically see their patients more frequently and should reiterate medication adherence. Establishing clear guidelines in collaboration with other healthcare practitioners on how to properly administer and take opioid medication is important to prevent OD.

Athletic training as a profession is already considered an aid to improving public health, by providing health services to various patient populations. ${ }^{15}$ To assist in the public health sector on opioid use, athletic trainers should focus on patient, organization member, and stakeholder education for prevention strategies and crisis response, as they do for other high-risk injuries like concussion management for athletes, coaches, and parents. Effective policy development and implementation on opioid use and OD prevention will help reinforce athletic training as a valuable Volume 4 - Issue 2 - September 2021 
allied health profession. This systematic review supported the feasibility and benefits of implementing $T H N$ to reverse opioid $O D$ and should be considered by all athletic trainers due to its benefits to patients and the community

\section{CLINICAL BOTTOM LINE}

Overall, THN programs were found to be effective in reducing deaths from opioid-induced ODs. We recommend that athletic trainers include opioid crisis management equipment and procedures in a site-specific policies manual. Naloxone administration may be compared to epinephrine-injections used for anaphylaxis or AED and CPR for cardiac emergencies. More than $90 \%$ of the ODs witnessed (and reversed) in the review were heroin-induced, therefore clinical relevance is highly dependent on patient population and geographic location. Recommendations from this review do not address applicability for individuals involved in organized sport where substances are regulated by a governing body.

The authors suggest individuals in physically active settings who are prescribed opioids for pain management should be candidates for THN programs, particularly in areas highly affected by the opioid epidemic. Primary preventative screening using self-report questionnaires (e.g. Opioid-Related Overdose Risk Behavior Scale [ORBS] ${ }^{16}$ or Alcohol, Smoking and Substance Involvement Screening Test [ASSIST] ${ }^{17}$ ) should be implemented by or in collaboration with prescribing physicians to assess patient need for THN. Prior OD risk screening may be especially useful if THN programs are not feasible for the patient due to socioeconomic factors and/or availability. If opioid medication is prescribed, patients should be thoroughly educated on medication adherence. Guardians of minors prescribed opioids should be educated and encouraged to dispense the medication as instructed by a physician or pharmacist. Athletic

Copyright (C) by Indiana State University All rights reserved. ISSN Online 2577-8188 trainers involved in the care of individuals prescribed opioid medications should be educated on best practices for opioid crisis management and aware of state legislature regarding administration and distribution of naloxone/THN programs. If improvements in athletic training education and professional development on opioid use are executed, then the public may see improved patient safety and positive community-based responses.

\section{REFERENCES}

1. Ahmad FB, Escobedo LA, Rossen LM, Spencer $M R$, Warner $M$, Sutton P. Provisional drug overdose death counts. National Center for Health Statistics. 2020. https://www.cdc.gov/nchs/nvss/vsrr/drugoverdose-data.htm.

2. Scholl L, Seth P, Kariisa M, Wilson N, Baldwin G. Drug and opioid-involved overdose deaths-United States, 2013-2017. Morbidity and Mortality Weekly Report. 2019;67(5152): 1419. http://dx.doi.org/10.15585/mmwr.mm675 $152 \mathrm{e} 1$.

3. McDonald R, Strang J. Are take-home naloxone programmes effective? Systematic review utilizing application of the Bradford Hill criteria. Addiction. 2016;111(7):11771187. https://doi.org/10.1111/add.13326.

4. World Health Organization. Community management of opioid overdose. https://www.who.int/publications/i/item/97 89241548816. 2014.

5. Strang J, Darke S, Hall W, Farrell M, Ali R. Heroin overdose: the case for take-home naloxone. BMJ. 1996;312:1435. https://dx.doi.org/10.1136\%2Fbmi.312.70 44.1435 .

6. Office of the Surgeon General. Surgeon General's Advisory on Naloxone and Opioid Overdose. In: Services UDoHaH, ed2018. https://www.hhs.gov/surgeongeneral/priorit 
ies/opioids-and-addiction/naloxoneadvisory/index.html.

7. Veliz PT, Boyd C, McCabe SE. Playing through pain: sports participation and nonmedical use of opioid medications among adolescents. Am $J$ Public Health. 2013;103(5):e28-e30. https://dx.doi.org/10.2105\%2FAJPH.2013. 301242.

8. McDuff D, Stull T, Castaldelli-Maia JM, Hitchcock ME, Hainline B, Reardon CL. Recreational and ergogenic substance use and substance use disorders in elite athletes: a narrative review. $\mathrm{Br} J$ Sports Med. 2019;53(12):754-760.

https://doi.org/10.1136/bjsports-2019100669.

9. Lucas RM, McMichael AJ. Association or causation: evaluating links between "environment and disease". Bull World Health Organ. 2005;83:792-795.

10. Walley AY, Xuan Z, Hackman HH, et al. Opioid overdose rates and implementation of overdose education and nasal naloxone distribution in Massachusetts: interrupted time series analysis. BMJ. 2013;346:f174. https://doi.org/10.1136/bmi.f174.

11. Coffin PO, Sullivan SD. Cost-effectiveness of distributing naloxone to heroin users for lay overdose reversal. Ann Intern Med. 2013;158(1):1-9. https://doi.org/10.7326/0003-4819-1581-201301010-00003.

12. Coffin PO, Sullivan SD. Cost-effectiveness of distributing naloxone to heroin users for lay overdose reversal in Russian cities. J Med Econ. 2013;16(8):1051-1060. https://doi.org/10.3111/13696998.2013. 811080.

13. Rowe C, Santos GM, Vittinghoff E, Wheeler E, Davidson P, Coffin PO. Predictors of participant engagement and naloxone utilization in a community-based naloxone distribution program. Addiction. 2015;110(8):1301-1310. https://doi.org/10.1111/add.12961.
14. Seal KH, Thawley R, Gee L, et al. Naloxone distribution and cardiopulmonary resuscitation training for injection drug users to prevent heroin overdose death: a pilot intervention study. J Urban Health. 2005;82(2):303-311. https://doi.org/10.1093/iurban/iti053.

15. Hoffman $M$, Bovbjerg $V$, Hannigan $K$, et al. Athletic training and public health summit. $J$ Athl Train. 2016;51(7):576-580. https://dx.doi.org/10.4085\%2F10626050-51.6.01.

16. Pouget ER, Bennett AS, Elliott $L$, et al. Development of an opioid-related overdose risk behavior scale (ORBS). Subst Abus. 2017;38(3):239-244. https://doi.org/10.1080/08897077.2017. 1282914.

17. World Health Organization. The ASSIST project-Alcohol, Smoking and Substance Involvement Screening Test. https://www.who.int/publications/i/item/97 8924159938-2. 2010. 